\title{
Donación cadavérica y trasplantes de órganos en México. Análisis de 12 años y siete propuestas estratégicas
}

\author{
Deceased donation and organ transplantation in Mexico. Analysis of 12 years and seven \\ strategic proposals
}

\author{
Rubén Argüero-Sánchez * , Omar Sánchez-Ramírez y Enrique M. Olivares-Durán²

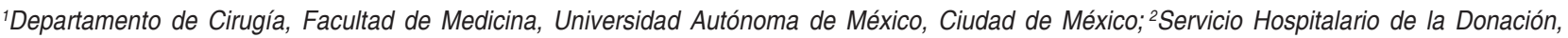 \\ Unidades Médicas de Alta Especialidad, Hospital de Especialidades N.o 1, Instituto Mexicano del Seguro Social, Centro Médico Nacional del Bajío, \\ León, Guanajuato. México
}

\begin{abstract}
Resumen
Antecedentes: Se analiza la evolución de la actividad de donación cadavérica de órganos y de la realización de trasplantes de órganos sólidos en México de 2007 a 2018. Método: Se recolectó y ordenó la información de donación cadavérica y de trasplante de órganos en México en ese período. Cuando no hubo información completa de 2018, se consideró la de 2017. Las principales fuentes de información fueron The Global Observatory on Donation and Transplantation y el Centro Nacional de Trasplantes de la Secretaría de Salud de México. Resultados: 1) La tasa de donación cadavérica de México en 2007 fue de 3.2 donaciones por millón de población (pmp), y en 2017 fue de 3.94 pmp (incremento del 23.1\%). La tasa de 2017 está muy por debajo de la media reportada para América Latina (9.5 pmp). 2) El número de pacientes en espera de trasplante de un órgano sólido pasó de 4,993 en 2007 a 15,448 en 2018 (incremento del 309\%). 3) En 2017, México reportó tener 255 centros autorizados para trasplante renal, más que cualquier país en el mundo. Para el caso de otros órganos, como hígado, corazón, pulmón y páncreas, México es el país que tiene más centros autorizados en América Latina. Conclusión: La evolución de las cifras de donación cadavérica y de trasplante de órganos sólidos en el período de estudio explica una escasez cada vez mayor de órganos para trasplante en México, a pesar de una aparente gran capacidad instalada. El sistema nacional de donación y trasplante de órganos es ineficaz e ineficiente.
\end{abstract}

Palabras Clave: Donación cadavérica de órganos. Trasplante de órganos. Problemática.

\section{Abstract}

Background: The evolution of the activity of deceased organ donation and solid organ transplantation in Mexico was analyzed for the period of 2004-2018. Method: The information of deceased donation and organ transplantation in Mexico was collected and ordered for said period. When there was no complete information for 2018, the data for 2017 were used instead. Main sources of information: The global observatory on donation and transplantation and the National Transplant Center, Centro National de Trasplantes or CENATRA of the Mexican Secretariat of Health (SSA). Results: (1) In Mexico, deceased donation rates were: 3.2 donations per million population (pmp) in 2007 and 3.94 in 2017, an increase of 23.1\%. The rate in 2017 is quite lower than the average reported for Latin America 9.5 donations pmp. (2) The number of patients

\footnotetext{
Correspondencia:

*Rubén Argüero-Sánchez

Bosque del Castillo, 82

Col. La Herradura

Fecha de recepción: 17-06-2019

Cir Cir. 2020;88(3):254-262

C.P. 82784, Huixquilucan, Edo. de México, México

Fecha de aceptación: 12-08-2019

ontents available at PubMed

E-mail: rubenarguero@gmail.com

DOI: $10.24875 / C I R U .19001379$

www.cirugiaycirujanos.com

0009-7411/@ 2019 Academia Mexicana de Cirugía. Publicado por Permanyer. Este es un artículo open access bajo la licencia CC BY-NC-ND (http://creativecommons.org/licenses/by-nc-nd/4.0/).
} 
awaiting a solid organ transplant increased from 4993 in 2007 to 15,448 in 2018, an increase of 309\%. (3) In 2017, Mexico reported having 255 authorized centers for renal transplantation, more than any other country in the world. In the case of other organs: liver, heart, lung, and pancreas, Mexico is the country with the highest number of authorized centers in Latin America. Conclusion: The evolution of the figures regarding the activity of deceased donation and solid organ transplantation during the aforementioned study period explains an increasing shortage of organs for transplants in Mexico, despite an apparent large installed capacity. The national organ donation and transplantation system is ineffective and inefficient.

Key Words: Deceased organ donation. Organ transplantation. Problematic.

\section{Introducción}

El 21 de julio de 1988 se realizó el primer trasplante de corazón en México'. A 31 años de este acontecimiento, este documento analiza la evolución y el estado actual de la actividad de donación cadavérica de órganos y de los trasplantes en México durante 12 años, de 2007 a 2018.

\section{Método}

Se recolectó y ordenó la información relacionada con la actividad de donación y trasplante de órganos en México, del año 2007 al 2018 inclusive. Cuando no se contó con toda la información oficial y pertinente del año 2018 se tomaron los datos hasta 2017. Las principales fuentes de información fueron:

- The Global Observatory on Donation and Transplantation (GODT; http://www.transplant-observatory.org/), que es un centro internacional auspiciado por la Organización Mundial de la Salud que cuenta con la colaboración de la Organización Nacional de Trasplantes de España. En su sitio electrónico se recopilan los datos de donación y trasplantes reportados por los organismos oficiales de cada país, y se presentan organizados por región, año, país, órgano, etc. Esta información es de acceso público.

- El Centro Nacional de Trasplantes (CENATRA; https://www.gob.mx/cenatra) de la Secretaría de Salud de México, a través de su página web.

En este artículo, el análisis de la donación cadavérica de tejidos y el tema de los donadores vivos de órganos serán abordados solo de manera marginal.

En primer término, se revisarán las cifras de donación de órganos en México y las comparaciones con otros países que se pueden hacer en este rubro. Luego se analizarán las cifras en el registro nacional de pacientes en espera de recibir un trasplante y los cambios que se han producido en el período de estudio. A lo largo del artículo se emplearán prefentemente las cifras enstandarizadas por millón de población (pmp), a través de tasas referidas a la población nacional o institucional, por ejemplo del Instituto Mexicano del Seguro Social (IMSS), cuando sea necesario, dado que esta es la forma más frecuente de expresar las cifras en este campo de la medicina en todo el mundo. Posteriormente se analizarán las cifras de trasplantes de órganos sólidos realizados, con énfasis en el trasplante renal, comparándolas con las de otros países latinoamericanos. Así mismo, se analizará la distribución de los trasplantes de órganos sólidos en general, y la de los trasplantes renales en particular, en el segmentado y multiinstitucional Sistema Nacional de Salud (SNS) de México. Como información extra se agregarán los datos correspondientes a los trasplantes de córnea, que siendo tejidos son históricamente el segundo trasplante más frecuente en nuestro país. Por último, se revisarán las cifras de la capacidad instalada en cuanto a número de centros hospitalarios autorizados para realizar trasplante de órganos sólidos en México, y se compararán con las de otros países.

\section{Resultados}

\section{Donación de órganos de fallecidos con diagnóstico de muerte encefálica}

En la figura 1 se muestra la evolución de la tasa nacional de donación entre 2007 y 2017. Dicha tasa fue en 2007 de 3.2 donaciones pmp y en 2017 se incrementó a 3.94 pmp $^{2,3}$ (incremento del 23.1\%). También en 2017, las tasas de donación cadavérica de algunos otros países de América Latina fueron: Argentina 13.4 pmp, Chile 9.6 pmp, Brasil 16.3 pmp, Colombia 8.9 pmp, Cuba 12.3 pmp y Uruguay 18.9 $\mathrm{pmp}^{2,3}$. América Latina, como región, tuvo en 2017 una tasa media de $9.5 \mathrm{pmp}^{4}$. Cifras aún más altas se 


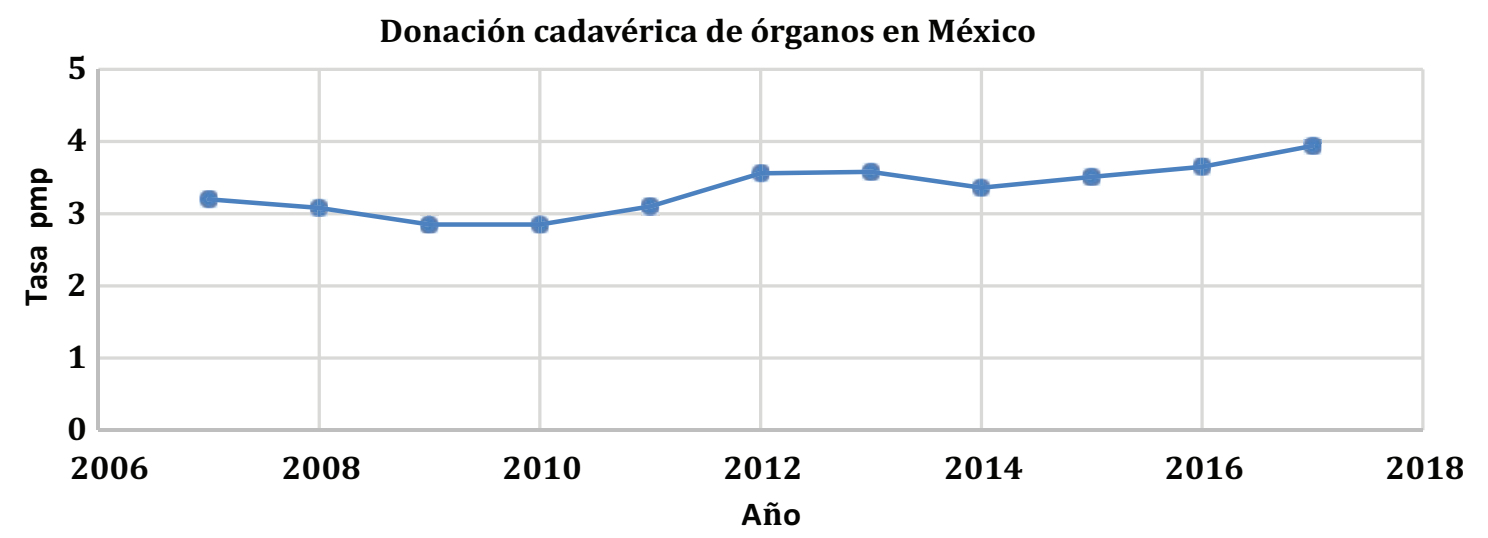

Figura 1. Tasa de donación cadavérica de órganos por millón de población (pmp) en México, 2007-20176.

Registro de pacientes en lista de espera para recibir un trasplante de órgano en México

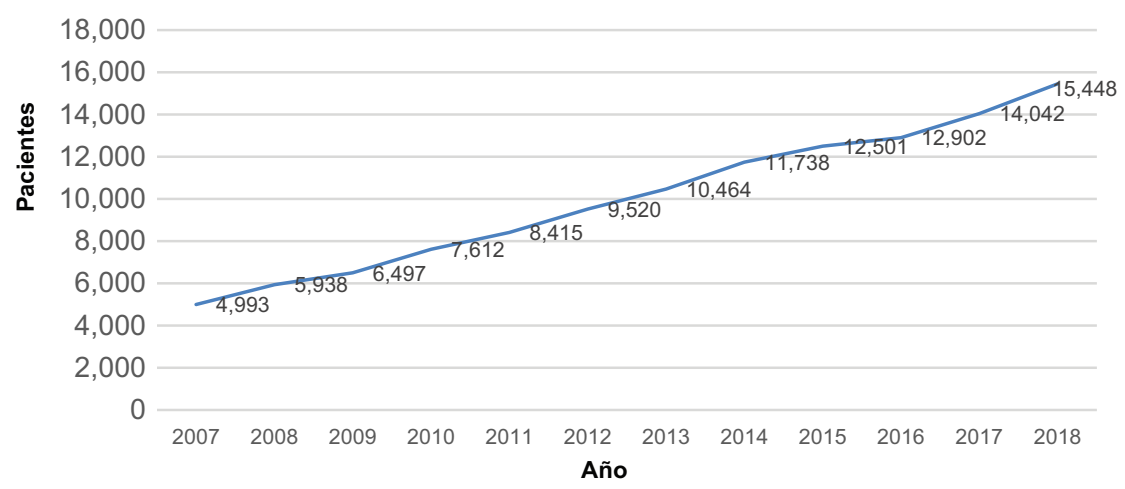

Figura 2. Número de pacientes en lista de espera por un órgano sólido en México, 2007-20186.

reportaron ese mismo año en dos de los países líderes en este campo, como los Estados Unidos, con una tasa de 31.7 pmp, y España, con una tasa de $47.0 \mathrm{pmp}^{2}$.

\section{Pacientes registrados en espera de recibir un trasplante de órgano}

La figura 2, elaborada a partir de datos publicados por el CENATRA ${ }^{5,6}$, muestra que el número de pacientes dados de alta en el Sistema Informático del Registro Nacional de Pacientes en espera de recibir un órgano sólido, sin considerar tejidos como la córnea, pasó de 4,993 pacientes en 2007 a 14,042 pacientes el año 2017 y 15,448 pacientes a finales de 2018, lo cual representa un incremento del $281 \%$ para el período $2007-2017$ y del 309\% para el período 2007 2018. Dicho incremento estuvo dado en su mayor parte por pacientes en espera de recibir un trasplante de riñón. Así, en 2007, el porcentaje de pacientes en el registro en espera de un riñón era del $91.8 \%$ con
Tabla 1. Trasplantes de órganos sólidos (riñón, hígado, corazón, pulmón, páncreas, intestino) en diversos países de América Latina en 2017. No se incluyen trasplantes de córnea y de otros tejidos ${ }^{3}$

\begin{tabular}{lcc}
\hline País & $\begin{array}{c}\text { Número total de trasplantes de } \\
\text { órganos sólidos de donadores } \\
\text { vivos y fallecidos en 2017 }\end{array}$ & $\begin{array}{c}\text { Tasa por millón } \\
\text { de habitantes (\%) }\end{array}$ \\
\hline Uruguay & 184 & 52.57 \\
Argentina & 1,999 & 45.12 \\
Brasil & 8,668 & 41.41 \\
Chile & 540 & 28.0 \\
Colombia & 1,330 & 27.09 \\
México & 3,386 & 26.21 \\
Cuba & 225 & 19.57 \\
\hline
\end{tabular}

respecto al número total de registros para todo tipo de órganos sólidos, es decir, sin contar la lista para córneas u otros tejidos, y para el año 2018 alcanzó un $97.6 \%$. 


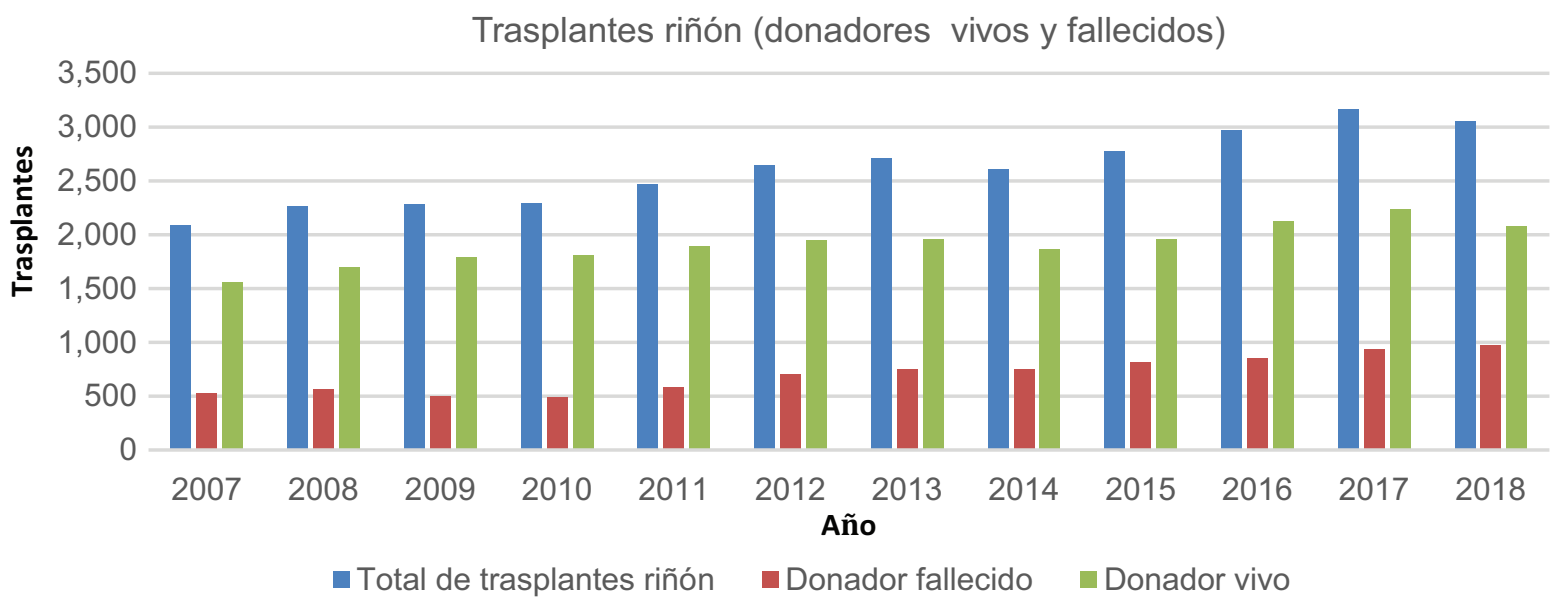

Figura 3. Número total anual de trasplantes de riñón en México y desglose según los procedentes de donador fallecido y de donador vivo, en el período 2007-2018

\section{Número de trasplantes de órganos sólidos realizados en diferentes países de América Latina en 2017 expresado en tasa por millón de habitantes}

En la tabla 1 se consignan los datos del número total de trasplantes de órganos sólidos, suma de los trasplantes de riñón, hígado, corazón, pulmón y páncreas reportados ese año, realizados en diversos países latinoamericanos. Se incluyen los órganos trasplantados y obtenidos tanto de donadores vivos como de donadores cadavéricos que se realizaron en México durante el año 2017 (tasa de 26.21 pmp) y se comparan con los realizados en otros países durante ese mismo año ${ }^{3}$.

\section{Trasplantes de riñón}

El número de trasplantes de riñón en nuestro país ha ido en aumento a lo largo de estos 12 últimos años, tal como se observa en la figura 3 , donde se muestran tanto las cifras totales anuales como los trasplantes procedentes de donante vivo y de donante fallecido ${ }^{6}$.

En la tabla 2 se realiza la comparativa entre diversos países latinoamericanos de la productividad en 2017 en trasplantes renales procedentes de donación cadavérica ${ }^{3}$. Si se consideran solo los números absolutos, México ocupó el segundo lugar, por debajo de Brasil y prácticamente con la misma productividad de Argentina. Sin embargo, cuando la comparación se realiza con las tasas pmp, México ocupa el último lugar entre los países comparados.
Tabla 2. Número total y tasa por millón de población de trasplantes de riñón procedentes de donación cadavérica realizados en 2017 en diversos países de América Latina ${ }^{3}$

\begin{tabular}{lcc}
\hline País & $\begin{array}{c}\text { Número de trasplantes } \\
\text { de riñón procedentes de } \\
\text { donadores fallecidos en 2017 }\end{array}$ & $\begin{array}{c}\text { Tasa por millón de } \\
\text { población (\%) }\end{array}$ \\
\hline Uruguay & 128 & 36.57 \\
Brasil & 4,807 & 22.97 \\
Argentina & 929 & 20.97 \\
Colombia & 798 & 16.25 \\
Chile & 292 & 16.13 \\
Cuba & 174 & 15.13 \\
México & 932 & 7.21 \\
\hline
\end{tabular}

\section{Distribución de los trasplantes renales en el SNS de México}

La atención a la salud en nuestro país esta fragmentada y es proporcionada por una amplia variedad de instituciones que, además, realizan donación y trasplantes de órganos. En la tabla 3 se presenta cómo se distribuye la derechohabiencia entre las instituciones que conforman el SNS, así como el número de trasplantes renales realizados por cada institución ${ }^{6}$ y la tasa pmp referenciada a su población derechohabiente durante el año 2018.

\section{Distribución de los trasplantes de córnea en el SNS de México}

Aunque este artículo tiene un enfoque centrado en el trasplante de órganos sólidos, en la tabla 4 se muestran 
Tabla 3. Distribución porcentual, en número total y en tasas por millón de población del total de los trasplantes de riñón (tanto de donador vivo como de donador fallecido), en México, entre las diversas instituciones del Sistema Nacional de Salud en $2018^{6}$

\begin{tabular}{lcccccc}
\hline & IMSS & Privado & ISSSTE & $\begin{array}{c}\text { PEMEX-Defensa- } \\
\text { Marina }\end{array}$ & $\begin{array}{c}\text { Seguro Popular o para } \\
\text { una Nueva Generación }\end{array}$ & $\begin{array}{c}\text { Total } \\
\text { nacional }\end{array}$ \\
\hline Distribución & $39.19 \%$ & $3.27 \%$ & $7.7 \%$ & $1.15 \%$ & $48.69 \%$ & $100 \%$ \\
Población expresada en millones & 48.88 & 4.08 & 9.61 & 1.43 & 60.74 & 124.74 \\
Número de trasplantes realizados & 1,513 & 615 & 108 & 48 & 364 & 3,048 \\
Tasa por millón de población & 31.0 & 150.8 & 11.2 & 33.5 & 12.6 & 24.4 \\
\hline IMSS: Instituto Mexicano del Seguro Social; ISSSTE: Instituto de Seguridady Servicios Sociales de los Trabajadores del Estado. & &
\end{tabular}

IMSS: Instituto Mexicano del Seguro Social; ISSSTE: Instituto de Seguridad y Servicios Sociales de los Trabajadores del Estado.

Tabla 4. Distribución de los trasplantes de córnea en las diversas instituciones del Sistema Nacional de Salud de México en el año 2018, en números totales y en tasas por millón de población ${ }^{6}$

\begin{tabular}{lcccccc}
\hline & IMSS & Privado & ISSSTE & $\begin{array}{c}\text { PEMEX-Defensa- } \\
\text { Marina }\end{array}$ & $\begin{array}{c}\text { Seguro Popular o para una } \\
\text { Nueva Generación }\end{array}$ & Nacional \\
\hline Número de trasplantes de córnea realizados & 1,523 & 2,066 & 121 & 49 & 510 & 4,269 \\
Tasa por millón de población & 31.2 & 506.4 & 12.6 & 34.3 & 8.4 & 34.2 \\
\hline
\end{tabular}

Tabla 5. Comparativa internacional del número de centros autorizados para realizar trasplantes de órganos sólidos por país en $2017^{2}$

Número de centros de trasplantes existentes autorizados por órgano

\begin{tabular}{lcccccc}
\hline País & \multicolumn{6}{c}{ Riñón Hígado Corazón Pulmón Páncreas $\begin{array}{r}\text { Intestino } \\
\text { delgado }\end{array}$} \\
\hline Uruguay & 3 & 1 & 3 & 1 & 1 & 0 \\
Brasil & 140 & 67 & 44 & 8 & 17 & 2 \\
Argentina & 53 & 29 & 22 & 4 & 17 & 2 \\
Colombia & 22 & 9 & 8 & 4 & 5 & 4 \\
Chile & 22 & 9 & 8 & 5 & 3 & 2 \\
Cuba & 9 & 3 & 1 & 0 & 0 & 0 \\
México & 255 & 73 & 51 & 10 & 22 & 3 \\
Estados & 240 & 144 & 138 & 71 & 135 & 40 \\
Unidos & & & & & & \\
España & 40 & 25 & 16 & 8 & 13 & 3 \\
\hline
\end{tabular}

los datos del año 2018 para la distribución interinstitucional de trasplantes de córnea ${ }^{6}$, que es un tejido. Este es el segundo trasplante más frecuentemente realizado en nuestro país, y los datos a él asociados agregan información relevante al panorama de la donación y el trasplante de órganos y tejidos en México.

\section{Capacidad instalada}

En el año 2017, México reportó contar con el mayor número de centros existentes autorizados para trasplante renal, incluso cuando se compara con los países líderes en este campo, como los Estados Unidos y España ${ }^{2}$, tal como se muestra en la tabla 5. Para el caso de otros órganos, como hígado, corazón, pulmón y páncreas, nuestro país cuenta con más centros autorizados que cualquier otro de los países latinoamericanos.

\section{Discusión}

\section{Sobre los trasplantes de órganos sólidos}

1) Sobre la ineficacia del sistema de trasplantes de órganos en México

En 2017, México tuvo una tasa de trasplantes de órganos sólidos (procedentes tanto de donadores vivos como de donadores fallecidos) de $26.21 \mathrm{pmp}$, solo superior, entre los países latinoamericanos comparados en este artículo, a la de Cuba (19.57 pmp). Cuando comparamos nuestra capacidad instalada con la de otros países de América Latina, resulta que México tiene el mayor número de centros existentes autorizados para trasplante renal. Incluso cuando se compara con países más allá de Latinoamérica sorprende que sea mayor y supere a países líderes en el mundo, como los Estados Unidos y España. En el caso de otros órganos, como corazón, hígado, pulmón y páncreas, México cuenta con más centros autorizados que cualquier otro de los países latinoamericanos; pese a ello, la mayoría nos superan en productividad. Es más que obvio que en nuestro país 
tenemos un grave problema tanto de eficiacia como de eficiencia en el tema de los trasplantes de órganos sólidos: una capacidad instalada supernumeraria con respecto a otros países acompañada de una baja productividad medida en tasa pmp. ¿Por qué? Muy probablemente se trata de un problema multifactorial cuyo análisis completo excede las capacidades de este artículo. Abordaremos, solo para empezar, una faceta del problema: es notorio que gran parte de la actividad de trasplante en riñón, hígado y corazón en México se concentra a lo mucho en 5-10 hospitales, tal como informa el CENATRA. La contribución que hacen todos los demás hospitales autorizados resulta desproporcionadamente baja. Así, por ejemplo, en 2018, el CENATRA informó que se realizaron 969 trasplantes de riñón de donante fallecido por muerte encefálica; de estos, 310 (32\%) fueron realizados en cinco hospitales ${ }^{6}$. Como resulta que hasta diciembre de 2017 México contaba con 255 centros autorizados para trasplante renal ${ }^{2}$, ello quiere decir que 250 hospitales realizaron los 659 trasplantes renales restantes $(68 \%)$ de todos los trasplantes renales de donante fallecido, un promedio de 2.6 trasplantes renales por año de donante fallecido, ipara cada uno de estos 250 hospitales! Obviamente las cosas no son así, sino más graves. Un número indefinido de hospitales no tiene nada que reportar, mientras que otros se distribuyen en pequeñas cantidades los 659 trasplantes. No es difícil imaginar que la experiencia quirúrgica y los resultados de estos trasplantes se vean comprometidos cuando la actividad es tan escasa y errática en muchos de los centros autorizados. ¿Por cuántos años debería mantenerse vigente la autorización para funcionar a un centro de trasplantes cuya productividad es nula o casi nula?

2) Distribución de los trasplantes de riñón en el SNS de México

Si volvemos a los datos presentados en la tabla 3 y ponemos atención en la tasa de trasplantes realizados en las diferentes instituciones del SNS mexicano en 2018, destaca que los hospitales privados alcanzan la tasa mayor (150.8 pmp), contra las tasas menores del sistema correspondientes al Seguro Popular (12.6 pmp) y al Instituto de Seguridad y Servicios Sociales de los Trabajadores del Estado (11.2 pmp); la tasa del IMSS es de $31.0 \mathrm{pmp}$, la tasa agrupada para PEMEX-Defensa-Marina es de $33.5 \mathrm{pmp}$ y la media de todo el SNS es de $24.4 \mathrm{pmp}$. De todos estos sectores, el más vulnerable por las condiciones socioeconómicas de sus afiliados es el correspondiente al Seguro Popular, que corresponde a cerca del $50 \%$ de la población nacional. La desproporción entre la tasa observada en 2018 de trasplante de órganos sólidos entre el sector privado y el Seguro Popular corresponde a una razón de 4:1. Esta desproporción se dispara aún más, hasta 60:1, cuando se hace la misma comparación entre hospitales privados y el Seguro Popular para el caso de los trasplantes de córnea realizados en México en 2018, mostrados en la tabla 4.

\section{Sobre la donación cadavérica de órganos y tejidos}

Pese a las actividades realizadas en el período de estudio (2007-2017), cuando se revisan las cifras del número de casos de donación de órganos de personas fallecidas bajo el diagnóstico de muerte encefálica se observa que el crecimiento ha sido sumamente pequeño, menor de 1 punto en la tasa de donación pmp, para alcanzar la cifra de 3.94 pmp en $2017^{2}$. Un reporte mexicano estima que en los últimos 40 años no ha habido un incremento significativo en las tasas de donación cadavérica ${ }^{7}$.

Este crecimiento mínimo en la tasa pmp de donaciones cadavéricas de órganos y tejidos contrasta con las cifras de otros países de América Latina durante el mismo período de tiempo, en el que Argentina, Chile, Brasil y Uruguay han alcanzado tasas muy superiores a las mexicanas, con una media para esta región algo más que el doble de la tasa en México.

Dicho estancamiento en las tasas de donación cadavérica de órganos en México se ha acompañado de un incremento del $281 \%$ en el número de pacientes en espera de recibir un órgano sólido en el período 2007-2017, mismo que sube hasta el $309 \%$ para el período 2007-2018 en dicha lista. Los autores de este artículo hicieron el ejercicio de convertir las cifras de las listas de espera a tasas pmp, y encontraron que la tasa pasó de 47.2 pmp en 2007 a 108.7 pmp en 2017, con un incremento del $230 \%$.

Ante estas bajas tasas de donación de órganos de pacientes fallecidos en México y la concomitantemente cada vez más acentuada escasez de órganos para trasplantes, ¿hacia dónde deberían encaminarse los esfuerzos en donación cadavérica de órganos? En nuestro país se ha impulsado la donación de órganos desde tres áreas: a) como un problema de índole cultural; b) como un tema de legislación; y c) como una tarea de capacitación. Mucho es lo que se ha escrito sobre estas tres importantes áreas, que no parecen 
ser, por lo menos en el sentido habitual con que se abordan, los eslabones más débiles en la actualidad en la cadena de procesos vinculados a la donación de órganos y tejidos en México. Las campañas constantes en pro de la donación cadavérica de órganos y tejidos, la evolución de la actitud de la sociedad mexicana, la de los medios de difusión y la del personal del SNS en México hacia este tema, parecen razonablemente proclives a favorecer la donación más que a combatirla o dificultarla. La legislación mexicana en la materia, si bien es perfectible, en general ampara, legitima y ordena la donación y los trasplantes en nuestro país, y algunas inquietudes recientes sobre si debemos contar con una legislación de donación presunta no parecen corresponder con los tiempos actuales en México, y tampoco con el modelo más exitoso en todo el mundo, que es el modelo español con su Organización Nacional de Trasplantes, en torno al cual se han alineado la mayoría de los países de América Latina, incluido México, y en el que, pese a tener una legislación de donación presunta, en la práctica se sigue prefiriendo contar con la anuencia-consentimiento de la familia ${ }^{8}$ para proceder a la donación cadavérica de órganos. Así mismo, la capacitación del personal que la Ley General de Salud en México autoriza y legitima para coordinar y ejecutar las labores esenciales del proceso de donación cadavérica de órganos, es decir, el coordinador hospitalario de la donación ${ }^{9}$, está sustentada por un diplomado universitario CENATRA/UNAM para la Formación de Coordinadores Hospitalarios de Donacion de Organos y Tejidos con fines de Trasplante $^{10}$, que no solo es obligatorio para completar el perfil requerido por ley l,11 $^{9}$ para acceder al puesto de coordinador hospitalario de la donación, sino que razonablemente se ha consituido en una herramienta educativa y de capacitación en el puesto muy eficiente y prestigiada por su calidad y mejora continua.

En resumen, en la coyuntura crítica de las estancadas tasas de donación en un nivel en el que son cada vez más insuficientes ante la escasez de órganos y tejidos en nuestro país, nos permitiremos realizar algunas sugerencias en campos en los que tenemos amplias áreas de oportunidad que no se han abordado, o al menos no como prioridades:

Propuesta 1: creación de una entidad de carácter público encargada de financiar los procesos de donación y trasplante de órganos y tejidos en México, alentando la productividad. Este ente administrativo se encargaría, entre otras tareas, de crear, regular y aplicar mecanismos de: a) reembolso de los gastos inmediatos en los que incurre un hospital durante los procesos de donación y trasplantes; b) compensación económica, bajo reglas claras y transparentes, de tiempo extra, fuera de horario de trabajo, al personal que participa en los procesos de donación, procuración y trasplante de órganos; y c) reembolso institucional (por hospital) de los costos de la inmunosupresión de los pacientes receptores de trasplantes.

Propuesta 2: convertir en letra viva la Declaración de Santiago de Querétaro del Colegio Mexicano de Medicina Crítica $^{12}$, y crear los indicadores y procesos de mejora y de incentivos para que los potenciales donantes cadavéricos de órganos sean ingresados con oportunidad y con carácter prioritario a las unidades de cuidados intensivos ( $\mathrm{UCl}$ ) y se atiendan en su inmensa mayoría dentro de las $\mathrm{UCI}$ mexicanas. Crear una guía de práctica clínica mexicana del manejo del paciente potencial donador cadavérico de órganos y tejidos en los servicios de urgencias y en las UCl. En resumen, normar y favorecer el rol relevante de las $\mathrm{UCl}$ en el proceso de atención del potencial donador, como ocurre por ejemplo en España, donde todos los procesos en que participan las $\mathrm{UCl}$ y los médicos intensivistas están incluidos y sujetos a recomendaciones específicas de la Guía de buenas prácticas en el proceso de la donación de órganos de la Organización Nacional de Trasplantes ${ }^{8}$.

Propuesta 3: Crear un programa mexicano de donación de órganos y tejidos en asistolia y convertirlo en una modalidad real y frecuente en México de donación cadavérica de órganos y tejidos, con impacto significativo en la tasa de donación pmp. Como dato que ilustra la relevancia potencial para México en el futuro de este programa, en el futuro de este programa en el Newsletter del GODT de $2018^{2}$, con las cifras definitivas del año 2017, de 66 países analizados, 22 reportaron actividad de donación en asistolia y ocho de ellos tuvieron tasas superiores a $4.0 \mathrm{pmp}$, con un rango de 4.6 pmp en Suiza a 12.3 pmp en España; estas cifras son totalmente independientes de las correspondientes a la actividad de donación cadavérica por muerte encefálica en esos países ${ }^{2}$. En este reporte, ningún país de América Latina aparece con actividad de donación en asistolia en 2017. Los reportes de nuestro país de donación en asistolia son escasos y ocasionales; así, en el período 2007-2017, en los informes anuales del GODT solo encontramos dos casos de donación de este tipo reportados por México el año 2012.

Propuesta 4: promover la creación de una Subcomisión de Donación y Trasplante de Órganos y Tejidos dentro de la Comisión de Salud de la Cámara de 
Diputados, con la que los médicos y otros profesionistas expertos en la materia puedan llevar una agenda formal y programática de corto, mediano y largo plazo, y que permita realizar los ajustes y las reformas legislativas que vayan siendo necesarios para las tres propuestas anteriores, y muchas otras que ya se han discutido en diversos foros, pero que no encuentran terreno propicio para prosperar en lo legislativo o en lo presupuestal.

Sugerimos brevemente algunas propuestas más, pero subrayando que la lista de estas no estará completa sin abrirse a la participación de todos los que viven profesionalmente la realidad de la donación y de los trasplantes de órganos y tejidos en México.

Propuesta 4: garantizar que los pacientes receptores de trasplantes dispongan de los medicamentos inmunosupresores que requieren.

Propuesta 5: crear las reformas legales requeridas para autorizar que las ambulancias de las diversas instituciones de salud puedan transportar y trasladar pacientes con muerte encefálica en los diversos procesos y subprocesos de la donación y trasplante de órganos, ya que los pacientes con muerte encefálica legalmente ya han perdido la vida y las ambulanicas no tienen permitido el traslado de cadáveres.

Propuesta 6: declarar oficialmente dentro de todo el SNS en México a los programas de donación y trasplantes como altamente prioritarios en todas las instituciones del sistema, y crear el marco legal y administrativo para tal efecto y para que el apoyo interinstitucional (SSA, IMSS, ISSTE, Centros Estatales de Trasplantes, etc.) esté debidamente reglamentado con el fin de lograr que en el ámbito local o regional se completen los procesos, para eficientar los recursos y disminuir los costos y los tiempos, y elevar la productividad en donación y trasplante de órganos y tejidos.

\section{Conclusiones}

La evolución de las cifras de donación y de trasplantes de órganos sólidos en el período 2007-2018 se ha caracterizado por: 1) un crecimiento exiguo, menor de 1 punto en la tasa pmp de donación cadavérica de órganos en México; 2) un incremento al triple, en números redondos, de la lista de pacientes en espera de un trasplante de un órgano sólido; y 3) una capacidad instalada en número de centros autorizados para trasplantes renales mayor que la de cualquier otro país en el mundo, y un número de centros autorizados para trasplantar hígado, corazón, pulmón y páncreas superior al de cualquier otro país latinoamericano, lo cual, ante la baja productividad y la mayor escasez de órganos, hace el diagnóstico de un sistema nacional de trasplantes no solo ineficaz, sino también ineficiente.

El sistema mexicano de donación y trasplantes de órganos es muy activo en números absolutos, pero insuficiente en tasas pmp para satisfacer las enormes necesidades de los pacientes en espera de un órgano. Urge un cambio estratégico integral en donación y trasplantes de órganos en México, que los autores de este artículo proponen que incluya, entre otras estrategias, un mayor presupuesto para la donación y trasplantes y una nueva manera de invertirlo, así como la promoción de una mayor participación de las $\mathrm{UCl}$ en la atención del potencial donador, y finalmente la implementación de un plan nacional de donación cadavérica en asistolia. Todos estos temas propuestos ameritan cada uno por sí solo una amplia discusión, de alcance nacional, por los expertos en la materia.

\section{Conflicto de intereses}

Los autores de este artículo afirman no tener conflicto de interés en la elaboración del mismo, para la cual no recibieron algún fondo o apoyo económico de alguna institución del sector público, privado, comercial, o de alguna ONG.

\section{Responsabilidades éticas}

Protección de personas y animales. Los autores declaran que para esta investigación no se han realizado experimentos en seres humanos ni en animales.

Confidencialidad de los datos. Los autores declaran que en este artículo no aparecen datos de pacientes.

Derecho a la privacidad y consentimiento informado. Los autores declaran que en este artículo no aparecen datos de pacientes.

\section{Bibliografía}

1. Argüero-Sánchez R. Trasplante de corazón. Rev Invest Clin. 2005;57:344-9.

2. European Directorate for the Quality of Medicines \& Health Care of the Council of Europe (EDQM) \& Organización Nacional de Trasplantes (ONT). International figures on donation and Transplantation 2017. Newsletter Transplant EDQM. 2018;23:1-66.

3. Red/Consejo Iberoamericano de Donación y Trasplante. Newsletter Trasplante Iberoamérica. 2018;12:1-78.

4. Organización Nacional de Trasplantes. Ministerio de Sanidad, Consumo y Bienestar Social. Gobierno de España. Gabinete de Prensa. Nota de Prensa. 20180829 Datos Registro Mundial de Trasplantes. 29 de agosto de 2018. (Consultado el 11 de julio de 2019.) Disponible en: http:// www.ont.es/prensa/NotasDePrensa/2018\%2008\%2029\%20\%20 DATOS\%20REGISTRO\%20MUN-DIAL\%20DE\%20TRASPLANTES.pdf 
5. Centro Nacional de Trasplantes. Reporte anual 2017 de donación y trasplantes en México. 2017. (Consultado el 3 de abril de 2019.) Disponible en: https://www.gob.mx/cms/uploads/attachment/file/289636/Presentaci_n_anual_2017.pdf

6. Centro Nacional de Trasplantes. Reporte anual 2018 de donación y trasplantes en México. 2018. (Consultado el 3 de abril de 2019.) Disponible en: https://www.gob.mx/cms/uploads/attachment/file/427652/Presentacion_anual_2018.pdf

7. Reyes-Acevedo R, Obrador GT, Alberú-Gómez J, Gracida-Juárez C, Madrigal JA, Aburto-Morales S. Current state and challenges for organ donation and transplantation in Mexico. Transplantation. 2019; 103:648-50.

8. Organización Nacional de Trasplantes (ONT). Guía de buenas prácticas en el proceso de la donación de órganos. Madrid: Gobierno de España. Ministerio de Sanidad, Política Social e Igualdad. ONT; 2011. p. 1-65.
9. Gobierno de los Estados Unidos Mexicanos. Ley General de Salud. Artículo 316-Bis. Diario Oficial de la Federación, 12-Dic-2011.

10. Centro Nacional de Trasplantes, CENATRA. Diplomado Universitario para la Formación de Coordinadores Hospitalarios de Donacion de Organos y Tejidos con fines de Trasplante. (Consultado el 16 de julio de 2019.) Disponible en: https://www.gob.mx/cenatra/acciones-y-programas/diplomado-universitario-para-la-formacion-de-coordinadores-de-donacion

11. Gobierno de los Estados Unidos Mexicanos. Reglamento de la Ley General de Salud en Materia de Trasplantes. Título Segundo, Capítulo II. Del Coordinador Hospitalario. Diario Oficial de la Federación, 26-Mar-2014.

12. Colegio Mexicano de Medicina Crítica (COMMEC). Declaración de Santiago de Querétaro sobre el proceso de donación y trasplante de órganos y tejidos de origen cadavérico en México. 13 de octubre de 2017. (Consultado el 16 de julio de 2019.) Disponible en: http://commec.org.mx/ images/declaracion-queretaro.pdf 